\title{
Hemoptisis recurrente como complicación de la fibrosis quística. Implicaciones para el tratamiento
}

\section{Recurrent hemoptysis as a complication of cystic fibrosis. Implications for treatment}

\author{
Jennifer Villar Zapata, MD.(1); Nelson lobelo, MD. (2); Catalina VÁsquez Sagra, MD. ${ }^{(3)}$; \\ JenNy JuRAdo HeRnándeZ, MD. ${ }^{(3)}$; AmAdor OVALLe, MD. ${ }^{(4)}$
}

\section{Resumen}

La hemoptisis es una complicación que se reporta comúnmente en los pacientes con diagnóstico de fibrosis quística y afecta aproximadamente al $9 \%$ de la población. Puede darse en escasa cantidad o en episodios masivos (4\%-5\% de los pacientes), definidos como sangrados superiores a $240 \mathrm{~mL}$ durante un periodo de 24 horas. Su patogénesis no está totalmente dilucidada, pero la causa más relacionada es la inflamación persistente de la vía aérea asociada a la neovascularización con tortuosidad y daño en la vasculatura de las arterias bronquiales, las cuales manejan presiones con valor sistémico.

Se describe el caso de un paciente joven con diagnóstico confirmado de fibrosis quística en quien se logró controlar la hemoptisis de manera adecuada a través de un tratamiento escalonado de la complicación.

Palabras clave: fibrosis quística, hemoptisis, embolización arterial, radiología intervencionista.

\section{Abstract}

Hemoptysis is a commonly reported complication in patients with cystic fibrosis. It affects about $9 \%$ of the population. It can be of scarce quantity or massive ( $4 \%-5 \%$ of patients); the latter is defined as bleeding greater than $240 \mathrm{~mL}$ during a 24 -hour period. Its pathogenesis is not completely understood, but the most commonly related cause is persistent inflammation of the airway associated with neovascularization, with tortuosity and damage of bronchial arteries, whose pressure values are systemic.

We describe the case of a young patient with confirmed cystic fibrosis in whom hemoptysis was successfully controlled with a stepwise treatment of the complication.

Keywords: cystic fibrosis, hemoptysis, arterial embolization, interventional radiology.

\section{Caso}

Paciente de 24 años, natural y procedente de Ibagué (Tolima), con diagnóstico de fibrosis quística a sus dos años de edad por síntomas digestivos persistentes y posteriormente respiratorios, con electrolitos en sudor positivos y estudio molecular que detectó mutación DF508. Ingresó al servicio de urgencias por cuadro clínico de una semana de evolución, conformado por astenia, adinamia, dificultad respiratoria, hemoptisis y deterioro de la función pulmonar; dentro de los últimos aislamientos microbiológicos se encontraban Staphylococcus aureus sensible a meticilina y Pseudomonas aeruginosa sensible a carbapenémicos. Desde el punto de vista radiológico se evidenciaron bronquiectasias difusas de predominio sacular hacia el lóbulo superior derecho (figura 1).
${ }^{(1)}$ Pediatra, Hospital Universitario Infantil de San José. Fundación Universitaria de Ciencias de la Salud. Bogotá, Colombia.

${ }^{(2)}$ Radiólogo Intervencionista, Hospital Universitario Infantil de San José.

(3)Neumólogo Pediatra, Fundación Neumológica Colombiana. Hospital Universitario Infantil de San José.

${ }^{(4)}$ Neumólogo Clínico. Grupo Multidisciplinario de Fibrosis Quística, Hospital Universitario Infantil de San José.

Correspondencia: Amador Ovalle. Correo electrónico: amadorovalle@gmail.com Recibido: 10-12-2013. Aceptado: 15-02-2014. 
Durante la hospitalización persistió la hemoptisis a pesar del tratamiento antibiótico, limitándolo para realizar la terapia respiratoria y su actividad física; dado el compromiso predominante del lóbulo superior derecho y la sospecha de que esta zona contribuía de manera importante a la hemoptisis, se propuso en junta médicoquirúrgica, con apoyo de Radiología intervencionista, realizar embolización arterial y evitar o posponer un tratamiento quirúrgico como la lobectomía.

Se hizo cateterismo de la arteria femoral y luego de la arteria bronquial que irriga el lóbulo superior derecho. Se hallaron múltiples vasos colaterales tortuosos; se embolizó con microesferas en dos viales y posteriormente se insertaron dos dispositivos tipo coil con los cuales se logró disminuir el sangrado.

Después del procedimiento evolucionó de manera satisfactoria, con notable disminución de los episodios de hemoptisis, tanto en número como en volumen de sangrado. Sin embargo, como efecto secundario cursó con dolor pleurítico por aproximadamente veinte días, sin otras complicaciones. Recibió tratamiento con polimixina $\mathrm{B}$ asociado a ciprofloxacina para cubrimiento de infección crónica por Pseudomonas aeruginosa multirresistente.

Siete meses después tuvo un nuevo episodio de hemoptisis masiva (100 mL en 24 horas), motivo

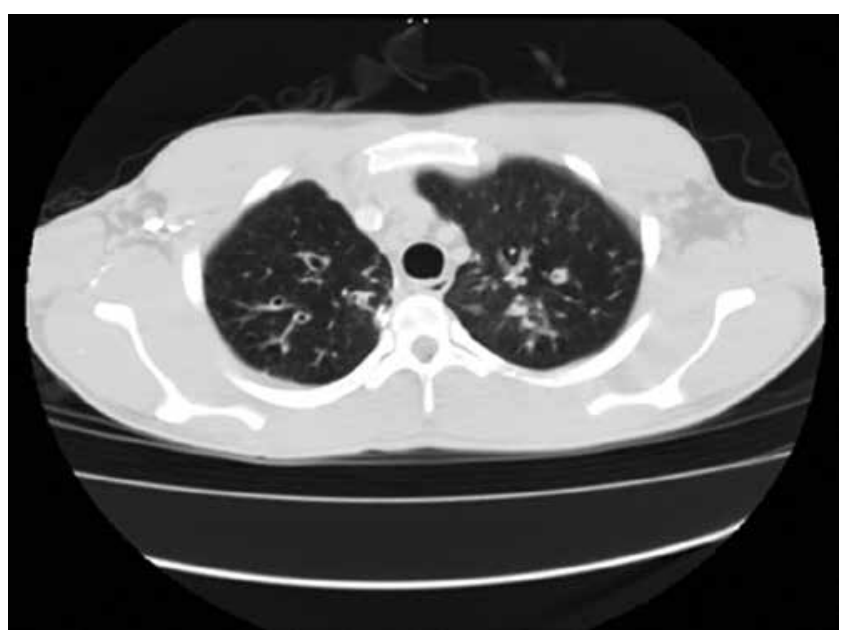

Figura 1. Tomografía de tórax que muestra bronquiectasias saculares en el lóbulo superior derecho. por el cual fue hospitalizado en su ciudad de origen, donde se inició antibioterapia biconjugada con el fin de controlar la infección endobronquial y ser remitido a una institución de mayor nivel de complejidad para posible manejo quirúrgico. Se realizó lobectomía superior derecha a través de toracoscopia, y desarrolló hemotórax en el postoperatorio inmediato, el cual se evacuó y controló con nueva toracoscopia y toracostomía derecha. La evolución posterior fue favorable. En la actualidad se encuentra en recuperación nutricional y de su condición física de manera progresiva, sin hemoptisis.

\section{Discusión}

La hemoptisis se define como el sangrado agudo de origen pulmonar, el cual puede ser masivo, esto es con un volumen de sangre de $240-300 \mathrm{~mL} /$ día, o menor en los casos recurrentes $(100 \mathrm{~mL} /$ día en 3 a 7 días); también se considera como todo sangrado que requiera transfusión de glóbulos rojos (1). La hemoptisis diaria de pequeñas cantidades puede

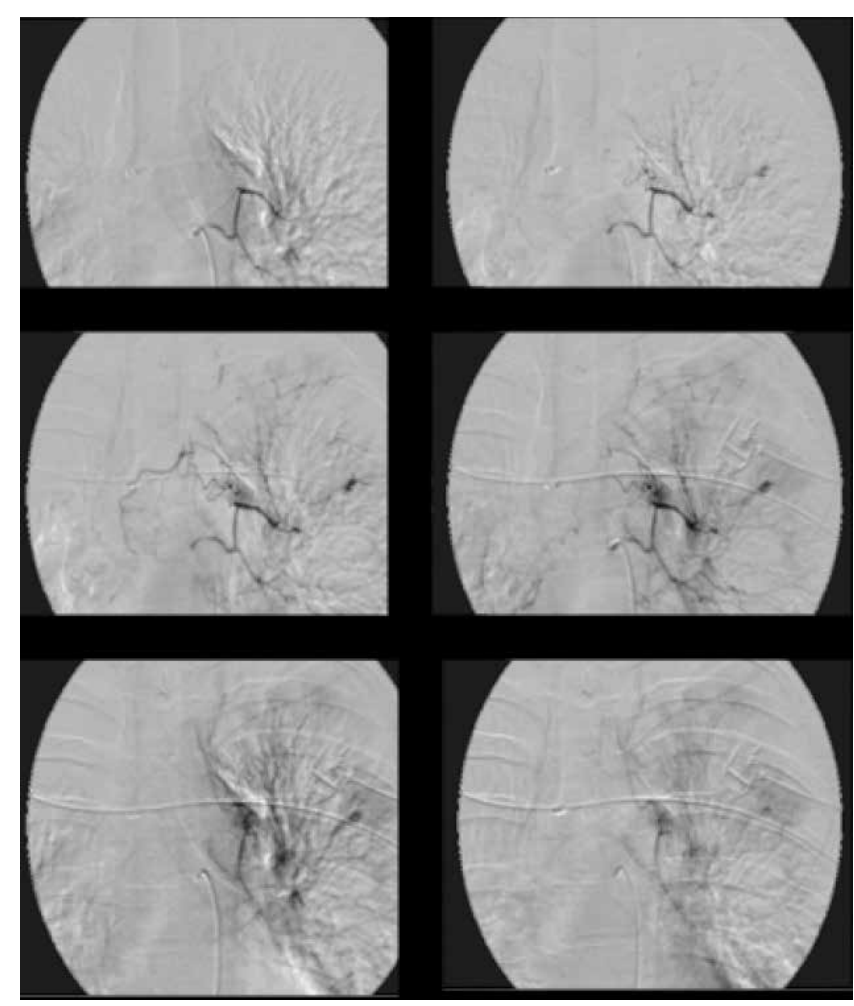

Figura 2. Cateterismo arterial y embolización selectiva de la arteria bronquial para el lóbulo superior derecho. 
convertirse en una complicación frecuente en los pacientes con fibrosis quística (2).

Es más común en adolescentes y rara en niños pequeños; se ha comprobado que se asocia con una mortalidad del $50 \%$ al $85 \%(3,4)$. Se estima que aproximadamente el 1\% de los enfermos con fibrosis quística presentará un episodio de hemoptisis masiva cada año, cuya frecuencia mayor se da entre aquellos que sobrepasan los 16 años de vida (2). El $22 \%$ de los pacientes cuentan con una función pulmonar normal (3); sin embargo, dentro de sus complicaciones más sobresalientes están la asfixia en el $80 \%$ y la anemia aguda por sangrado masivo en el $20 \%$ restante $(5,6)$.

\section{Pátogénesis}

El pulmón tiene doble sistema vascular, pulmonar y bronquial, y entre éstos pueden surgir múltiples anastomosis de predominio en los lechos capilares, que se encargan del intercambio gaseoso alveolar; la circulación bronquial es la responsable del 5\% del gasto cardiaco y está encargada de la irrigación de la estructura. La circulación bronquial maneja presiones sistémicas y es la responsable del $90 \%$ de los episodios de hemoptisis; puede, así mismo, generar sangrados masivos cuando cuenta con anastomosis con otras arterias como la mamaria interna, la subclavia, las intercostales y otras del orden sistémico, hecho que a su vez explicaría la importancia del tratamiento adecuado de los episodios de hemoptisis y las posibles complicaciones de la embolización (5, 6). Adicionalmente, se han demostrado procesos de neovascularización y remodelación vascular posteriores a procesos inflamatorios como bronquiectasias, bronquitis crónica o infecciones crónicas necrosantes del parénquima pulmonar adyacente a los bronquios, que contribuyen a la etiopatogenia de la hemoptisis $(5,6)$.

Esta condición tiene su origen en la erosión y posterior ruptura de una arteria bronquial con drenaje a la vía aérea, siendo la inflamación crónica de esta última la que estimula la hipertrofia y la neovascularización $(5,6)$. En los episodios agudos de exacerbación en los pacientes con fibrosis quística se han detectado niveles elevados del factor de crecimiento endotelial vascular (promotor de la angiogénesis); también se han identificado productos bacterianos que alteran el epitelio y el endotelio vascular y que estimulan la proliferación arterial bronquial (5), lo cual lleva a elongación, dilatación y tortuosidad de los vasos bronquiales, así como de sus anastomosis y sus colaterales (5-9).

$\mathrm{Su}$ etiología también puede verse enmarcada en otros orígenes como el tratamiento con fármacos antiinflamatorios no esteroides que interfieren con la hemostasia, el uso de fármacos irritantes en aerosol, el déficit de vitamina $\mathrm{K}$ y las variaciones en la presión arterial por cambios posturales (2). Aunque el diagnóstico de hemoptisis es clínico, la mayoría de los pacientes cuentan con un estudio imaginológico del tórax que permite determinar la severidad y extensión del compromiso parenquimatoso y de la pequeña vía aérea que puede acompañar su evolución (como en el caso de las bronquiectasias que pueden facilitar la presentación clínica inicial con expectoración hemoptoica o hemoptisis recurrente). En algunos casos conviene realizar fibrobroncoscopia para identificar con mayor precisión el sitio del sangrado. La evaluación complementa con el estudio de anormalidades en la coagulación como causa del sangrado (deficiencia de vitamina $K$, trastornos de la función hepática, trombocitopenia), antes de cualquier manejo específico (3).

Dentro de los diagnósticos diferenciales de hemoptisis se encuentran: los sangrados de la vía aérea superior o del tracto gastrointestinal, la aspiración de cuerpo extraño y la ingestión de medicamentos.

De otra parte, para el estudio de los casos de hemoptisis se investigan de manera integral sus probables causas y la condición del paciente, como mínimo con la ayuda de una radiografía de tórax, un hemograma (hemoglobina, hematocrito y plaquetas), pruebas de coagulación, hemoclasificación, pruebas de función hepática y muestras de secreciones bronquiales para estudio microbiológico (1).

Algunos episodios de hemoptisis pueden mejorar de forma espontánea sin necesidad de intervenciones o cambios en el tratamiento, mientras que otros, que sólo ocurren en los episodios de exacerbación, 
desaparecen luego del control de la misma, pero recidivan ante una nueva agudización (5-9).

Son estrategias del tratamiento habitual la administración de antibióticos de amplio espectro con óptima cobertura contra $S$. aureus y $P$. aeruginosa teniendo en cuenta que son más efectivos los manejos con medicación endovenosa que inhalada si bien su uso conjunto aumenta la efectividad (10-19, 22). Así mismo, figuran el aumento de las medidas de limpieza de la vía aérea como la utilización de alfa dornasa y la DNasa recombinante humana, que favorece el aclaramiento mucociliar al disminuir la viscosidad de las secreciones, y por ende los episodios de exacerbación con el fin de evitar infecciones severas (2).

El tratamiento de los episodios de hemoptisis comprende, de igual forma, un enfoque integral que incluye tranquilizar el paciente, brindar sedación eventual, garantizar el apoyo psicológico, suspender medicamentos potencialmente hemorragíparos o irritantes, ordenar suplencia de oxígeno, corregir el déficit de vitamina $\mathrm{k}$, transfundir ante pérdidas importantes de sangre e iniciar terapia con antibióticos, vasopresina, ácido tranexámico o desmopresina endovenosa $(2-11,12)$. Se recomienda evitar la percusión torácica durante 24 horas aunque no existe evidencia de que se deba suspender la terapia respiratoria; ésta debe reintroducirse lo antes posible para mejorar la higiene bronquial $(13,14)$. En los episodios de hemoptisis masiva o si se repite el sangrado (más de $100 \mathrm{~mL}$ diarios durante 7 días), se considera efectuar broncoscopia más embolización con el fin de localizar y controlar el sangrado, aunque se debe tener en cuenta que en ocasiones la hemorragia masiva impide una correcta visualización.

\section{Las indicaciones de embolización son:}

a) Hemoptisis persistente a pesar de otras opciones terapéuticas menos agresivas o que ponen en riesgo vital al paciente como para requerir una intervención inmediata.

b) Hemoptisis no masivas pero recurrentes y/o que interfieren con la calidad de vida del paciente (2).

Otras medidas terapéuticas son el taponamiento con balón del segmento o lóbulo sangrantes, la instilación de noradrenalina o el suero frío intrabronquial, aunque con una casuística menor de pacientes. Una de las posibles y eventuales complicaciones de la embolización es el infarto de órganos distales a la zona embolizada y la muerte. En casos graves que no respondan al tratamiento en mención, puede acudirse a la angiografía bronquial selectiva si persiste el sangrado. Podrá considerarse la lobectomía, luego de la embolización de las arterias sangrantes, en aquellos pacientes que no mejoran con la embolización y que cursan con sangrado desde un lóbulo plenamente identificado (1).

La embolización de arterias bronquiales es uno de los tratamientos más usados en hemoptisis de diferentes etiologías, incluyendo tuberculosis, bronquiectasias, aspergilosis, fístula aortobronquial y fibrosis quística. Con esta se logran disminuir las comorbilidades que se presentan al exponer a estos pacientes a tratamientos quirúrgicos (15); las principales contraindicaciones para embolización arterial incluyen: coagulopatía incorregible, falla renal y reacción alérgica severa a los medios de contraste (15-16).

Luego de la embolización arterial la tasa de recurrencia es de aproximadamente $50 \%$ en los cuatro primeros meses (4) y muchos casos requerirán nueva embolización (2), la cual puede llevarse a cabo hasta tres veces en el mismo episodio; luego se recurrirá a otras opciones terapéuticas si el sangrado perdura. La eficacia general para el control inicial de la hemoptisis después de una embolización es cercana al $75 \%$, aumenta al $89 \%$ después de dos embolizaciones y es del 93\% después de tres $(13,20-22)$.

La indicaciones para intervención quirúrgica en los pacientes con fibrosis quística se derivan principalmente de patologías pleurales como el neumotórax, el derrame pleural y el empiema; en cuanto a las alteraciones parenquimatosas están las bronquiectasias, la hemoptisis (con o sin bronquiectasias relacionadas) y los abscesos pulmonares, pero solo deben ser consideradas cuando los tratamientos médicos y las embolizaciones se hayan agotado en su totalidad. Entre las opciones sugeridas figuran la ligadura arterial selectiva y la lobectomía, aunque en cada una de estas es preciso tener en 
cuenta la capacidad pulmonar reducida del paciente y el riesgo de recurrencia de hemoptisis en otra localización (23).

En cuanto al trasplante pulmonar, las indicaciones actuales son el deterioro respiratorio progresivo demostrado por deterioro del VEF1 mayor del 30\%, la hipoxemia y la hipercapnia severas, el incremento en la frecuencia y duración de las exacerbaciones pulmonares que requieran hospitalización y la ausencia de respuesta a los manejos médicos como en el caso de hemoptisis masiva recurrente y resistencia bacteriana intratable (22).

\section{Conclusión}

La hemoptisis constituye una de las complicaciones esperadas en los pacientes que padecen fibrosis quística; generalmente es causada por hipertrofia de las arterias bronquiales en respuesta a anormalidades y lesiones repetidas o crónicas sobre el parénquima pulmonar con la respectiva erosión y ruptura de las mismas. El tratamiento antibiótico es el paso inicial de una serie de medidas escalonadas que requieren seguimiento clínico adecuado, durante el cual ocasionalmente se requiere embolización de las arterias bronquiales para estabilizar al paciente y obtener un excelente resultado a corto plazo y desenlaces variables a largo plazo; de igual manera, disminuye los riesgos y las complicaciones asociados con los tratamientos quirúrgicos, razón por la cual se continúa en la búsqueda de mayor precisión del momento para efectuar dicho procedimiento.

De otro lado, es importante optimizar la condición clínica del paciente antes de la intervención y procurar el diagnóstico correcto de la etiología de la hemoptisis, con el fin de ofrecer mejor calidad de vida y resultados favorables; la lobectomía se indica una vez haya falta de respuesta a las terapias habituales. En cualquier circunstancia es indispensable el seguimiento multidisciplinario que conduzca a obtener resultados óptimos.

\section{Bibliografía}

1. Consenso de fibrosis quística. Arch Argent Pediatr. 1999;97:188-224.

2. Prados C, Máiz L, Antelo C, Baranda F, Blázquez J, Borro JM, et al
Cystic fibrosis: consensus on the treatment of pneumothorax and massive hemoptysis and on the indications for lung transplantation. Arch Bronconeumol. 2000;36(7):411-6.

3. Flume PA. Pulmonary complications of cystic fibrosis. Respir Care. 2009;54(5):618-27.

4. Flume PA, Yankaskas JR, Ebeling M, Hulsey T, Clark LL. Massive hemoptysis in cystic fibrosis. Chest. 2005;128:729-738.

5. Charan NB, Baile EM, Pare PD. Bronchial vascular congestion and angiogenesis. Eur Respir J. 1997;10:1173-1180.

6. McDonald DM. Angiogenesis and remodeling of airway vasculature in chronic inflammation Am J Respir Crit Care Med. 2001;164:S39-S45.

7. Jewkes J, Kay PH, Paneth M, Citron KM. Pulmonary aspergilloma: analysis of prognosis in relation to haemoptysis and survey of treatment. Thorax. 1983;38:572-578.

8. Parker CM, Nolan R, Lougheed MD. Catamenial hemoptysis and pneumothoraces in a patient with cystic fibrosis. Can Respir J. 2007;14: 295-297.

9. McDonald DM. Angiogenesis and remodeling of airway vasculature in chronic inflammation Am J Respir Crit Care Med. 2001;164:S39-S45.

10. Emerson J, Rosenfeld M, McNamara S, et al. Pseudomonas aerugino$s a$ and other predictors of mortality and morbidity in young children with cystic fibrosis. Pediatr Pulmonol. 2002;34:91-100.

11. Barben JU, Ditchfield M, Carlin JB, Robertson CF, Robinson PJ,Olinsky A. Major haemoptysis in children with cystic fibrosis: a 20year retrospective study. J Cyst Fibr. 2003;2:105-111.

12. Popper J. The use of premarin IV in hemoptysis. Dis Chest. 1960;37:659-60.

13. Schidlow DV, Taussig LM, Knowles MR. Cystic Fibrosis Foundation consensus conference report on pulmonary complications of cystic fibrosis. Pediatr Pulmonol. 1993;15:187-98.

14. Flume PA, Mogayzel PJ Jr, Robinson KA, Rosenblatt RL, Quittell L, Marshall BC. Cystic fibrosis pulmonary guidelines: pulmonary complications: hemoptysis and pneumothorax. Clinical Practice Guidelines for Pulmonary Therapies Committee; Cystic Fibrosis Foundation Pulmonary Therapies Committee. Am J Respir Crit Care Med. 2010;182:298-306.

15. Coss-Bu JA, Sachdeva RC, Bricker JT, Harrison GM, Jefferson LS. Hemoptysis: a 10-year retrospective study. Pediatrics. 1997;100:E7.

16. Sidhu M, Wieseler K, Burdick TR, Shaw DW. Bronchial artery embolization for hemoptysis. Semin Intervent Radiol. 2008;25:310-8.

17. Barben J, Robertson D, Olinsky A, Ditchfield M. Bronchial artery embolization for hemoptysis in young patients with cystic fibrosis. Radiology 2002; 224:124-130.

18. Antonelli M, Midulla F, Tancredi G, Salvatori FM, Bonci E, Cimino G, Flaishman I. Bronchial artery embolization for the management of nonmassive hemoptysis in cystic fibrosis. Chest. 2002;121: 796-801.

19. Hurt K, Simmonds NJ. Cystic Fibrosis: Management of Haemoptysis. Paediatr Respir Rev. 2012; 13; 200-205.

20. Flume PA, Mogayzel Jr PJ, Robinson KA, et al. Cystic Fibrosis Pulmonary Guidelines: Pulmonary Complications: Hemoptysis and Pneumothorax. Am J Respir Crit Care Med 2010;182: 298-306.

21. Rolla M, D'Andrilli A, Rendina EA, Diso D, Venuta F. Cystic fibrosis and the thoracic surgeon. Eur J Cardiothorac Surg. 2011;39:716-25 Cystic fibrosis and the thoracic surgeon. Rolla M, D'Andrilli A, Rendina EA, Diso D, Venuta F.Eur J Cardiothorac Surg 2011;39:716-25.

22. Ryan G, Jahnke N, Remmington T. Inhaled antibiotics for pulmonary exacerbations in cystic fibrosis. Cochrane Database of Systematic Reviews 2012; 12: CD008319. 